\title{
L'iconographie de saint Yves et la politique dynastique des Montfort à la fin du Moyen Âge
}

Le 19 mai 1441, François, comte de Montfort, fils aîné du duc de Bretagne Jean V, confirmait la fondation d'une messe en l'honneur de saint Yves ainsi que de douze anniversaires établis par son père dans la cathédrale de Tréguier « pour la singuliere devocion quil avoit \& a au benoist glorieux confessoure mons. saint Yves $^{1}{ }^{\prime}$. Au-delà des conventions de la formule, le culte de saint Yves - patron des avocats et des gens de justice ${ }^{2}$, canonisé en 1347 - semble bien avoir revêtu pour Jean V et ses successeurs une importance toute particulière. Si le culte de l'official de Tréguier n'est pas resté limité au duché bien qu'il y fût plus important qu'ailleurs ${ }^{3}$, c'est au paysage culturel breton que semble appartenir essentiellement saint Yves, né vraisemblablement en 1253 à Kermartin (Côtes d'Armor) et mort le 19 mai 1303 à Laonnec (C.-d'A.).

En dépit de la très grande popularité du saint de Kermartin dans le duché et contrairement à ce que l'historiographie pourrait laisser croire, l'iconographie monumentale de saint Yves est relativement peu abondante en Bretagne à la fin du Moyen Âge. Présente parfois dans les porches bretons (Landivisiau, Bodilis, Kergist-Moëlou ou Guimiliau), les représentations du recteur de Trédrez restent essentiellement cantonnées à la sculpture mobiliaire, en grande partie postérieure au XVe siècle. L'iconographie judiciaire de saint Yves entre le riche et le pauvre plaideur constitue l'exemple le plus fréquent de ces représentations qui ne font aucune place aux épisodes de la vie du saint. L'absence « de retable, de volet, de niche consacrés à une imagerie narrative de la vie de saint $\mathrm{Yves}^{4} \gg$ a déjà été relevée ; le vitrail de l'église de Montcontour (Côtes-d'Armor) en 1537, venant tardivement combler ce vide documentaire.

\footnotetext{
${ }^{1}$ Archives départementales de Loire-Atlantique, E 83/14.

2 Ropartz (M. S.), Histoire de saint Yves, patron des gens de justice (1253-1303), Saint-Brieuc, L. Prud'Homme, 1856.

${ }^{3}$ Et dans le diocèse de Tréguier, plus important que dans le reste de la Bretagne (voir MinOIS (Charles), «Culte des saints et vie religieuse dans le diocèse de Tréguier au XVe siècle », dans Annales de Bretagne, 1980, 87, pp. $17-50 ;$ p. 42).

${ }^{4}$ DeBidour (Victor-Henri), La sculpture bretonne, Rennes, Ouest-France, 1981, p. 230.
} 
Jean-Marie Guillouët, «L'iconographie de saint Yves et la politique dynastique des ducs Montfort à la fin du Moyen Âge » ("Saint Yves' Iconografy and Dynastic Strategy in Late Medieval Britany »), in Annales de Bretagne et des Pays de l'Ouest, t. 107, 2000, pp. 23-40.

Pourtant, les portails de la façade de la cathédrale de Nantes recèlent un vaste cycle sculpté consacré à saint Yves qui n'a suscité que très peu d'intérêt jusqu'à présent. L'importance de ce cycle (vingt-six scènes conservées ; près d'une quarantaine à l'origine) ainsi que sa date (probablement dans la décennie 1450, soit presque exactement un siècle après la canonisation $\mathrm{du}$ saint) justifient pourtant qu'on s'y attache avec attention. Ce portail Saint-Yves, étroitement lié au pouvoir temporel du duc, manifeste-t-il une revendication culturelle bretonnante? Est-il l'expression d'une volonté politique plus spécifiquement dynastique? Bien que saint Yves soit traditionnellement regardé comme un saint breton, les sources disponibles le concernant ne semblent pas accorder à la dimension bretonne du personnage toute l'importance escomptée. Un détour par l'histoire mouvementée du duché au XIVe siècle et les querelles successorales qui opposèrent Charles de Blois et Jean de Montfort permettra de mettre en valeur toute l'ambiguité politique de l'iconographie du recteur de Trédrez. De manière paradoxale en effet, celle-ci trahit l'importation en Bretagne des pratiques monarchiques capétiennes.

\section{Le portail Saint-Yves de la façade nantaise}

Cinq portails, trois à l'ouest et deux en retour sous la travée des tours, forment la partie basse de la façade occidentale de la cathédrale de Nantes. Le cycle sculpté de saint Yves se trouve dans les voussures du portail latéral sud qui fait pendant au portail des saints Donatien et Rogatien, au nord. Trente-six scènes occupent les trois voussures de ce portail. On y repérera sans aucune difficulté les quelques groupes ayant fait l'objet d'un remplacement à l'époque contemporaine. Les quatre groupes inférieurs de la retombée est de la voussure extérieure ont été entièrement refaits, sans qu'il soit besoin d'y insister tant la facture en est grossière et maladroite. C'est au même sculpteur qu'il faut attribuer un groupe adjacent de la voussure intermédiaire. Hormis ces cinq scènes, l'ensemble ne montre aucune trace d'intervention récente et doit être considéré comme d'origine. Sur les trente et une scènes vierges de toute réfection, vingt-six seulement sont encore lisibles aujourd'hui que deux descriptions anciennes permettent de mieux comprendre ${ }^{5}$. A la lumière de ces descriptions et

\footnotetext{
${ }^{5}$ CAHOUR (abbé), « Iconographie et épigraphie de la cathédrale de Nantes par feu l'abbé Rousteau », dans Congrès Archéologique de France (Nantes), LIII ${ }^{\mathrm{e}}$, Paris, 1886, p. 207-243 et GABORIT (P.), Iconographie de la cathédrale de Nantes, Nantes, Bourgeois, 1892. Le texte de Gaborit n'est en fait qu'une paraphrase de la description antérieure de l'abbé Rousteau, transcrite par l'abbé Cahour, qu'on pourra se contenter de consulter.
} 
Jean-Marie Guillouët, «L'iconographie de saint Yves et la politique dynastique des ducs Montfort à la fin du Moyen Âge » (« Saint Yves' Iconografy and Dynastic Strategy in Late Medieval Britany »), in Annales de Bretagne et des Pays de l'Ouest, t. 107, 2000, pp. 23-40. au vu des scènes encore lisibles, il est possible de reconstituer le cycle de la manière suivante $^{6}$ :

Le cycle commence à la demi voussure intermédiaire est - à droite - du haut en bas : 1 «Maurice Dumont, sur l'ordre d'une voix céleste, se lève, pendant la nuit, et trouve saint Yves dans le cimetière, couché vêtu dans une concavité de rocher, où saint Elleau avait mené la vie pénitente. » $\mathbf{2}$ «Un pauvre passe la nuit à la porte du saint; il l'introduit chez lui, au matin, et lui laisse la disposition de sa maison, après l'avoir revêtu de ses propres habits. » $\mathbf{3}$ « Il couche, à la place de ce pauvre, à sa porte, pendant la nuit suivante. » $\mathbf{4}$ 《 Il demande au seigneur de Rostrenen la permission de prendre du bois dans ses forêts, pour faire la charpente de la cathédrale de Tréguier. » $\mathbf{5}$ « Il choisit les pieds d'arbres et les fait couper, et chaque pied en produisit, miraculeusement, trois à la place de celui qui était coupé. » 6 « Le bois est coupé et équarri, il s'allonge, miraculeusement, pour les besoins de l'édifice [moderne] ». Le cycle se continue ensuite à la demi voussure intérieure qui lui est adjacente, également de haut en bas : 1(7) « Saint Yves achète des pièces de drap pour vêtir des pauvres. » 2(8) « Il donne ses soins à un malade. » 3(9) «Il ensevelit un mort. » 4(10) Informe. 5(11) Informe. L'autre demi voussure intérieure, à l'ouest - gauche - reprend la suite de la narration, toujours de haut en bas : 1(12) « Le saint est endormi sur une chaise, qui était sa couche ordinaire. Un témoin le contemple. » 2(13) « Il donne à un pauvre un vêtement neuf qu'on lui apportait pour luimême. » 3(14) Sujet très défiguré. 4(15) « Saint Yves prêchant. » 5(16) « Saint Yves faisant le catéchisme à des enfants ». Puis vient la demi voussure intermédiaire du même côté, dans le même sens : 1(17) «Saint Yves, entrant dans une chaumière, y trouve un pauvre délaissé, qu'il fait transporter chez lui.» 2(18) «Saint Yves, soignant, de ces propres mains, ce malade. » 3(19) « Saint Yves passe, à pied sec, avec son domestique, une rivière dont il a séparé les eaux, par le signe de la croix. » 4(20) «Saint Yves tente de réconcilier un fils avec sa mère. » 5(21) «Saint Yves dit la messe à cette intention. » 6(22) «La réconciliation s'opère (réserve sur les trois dernières scènes qui sont très mutilées) ». Enfin, la demi voussure extérieure à l'ouest supporte les dernières scènes originales encore lisibles, du bas en haut semble-t-il cette fois-ci : 1(23) «Saint Yves étendu mort sur la claie; plusieurs personnages religieux sont arrêtés devant lui. » 2(24) «Hommages et supplications au tombeau de saint Yves. » 3(25) «Hommages et supplications au tombeau de saint Yves. »

\footnotetext{
${ }^{6}$ Cette description est pour une large part empruntée à celle de l'abbé Cahour.
} 
Jean-Marie Guillouët, «L'iconographie de saint Yves et la politique dynastique des ducs Montfort à la fin du Moyen Âge » («Saint Yves' Iconografy and Dynastic Strategy in Late Medieval Britany »), in Annales de Bretagne et des Pays de l'Ouest, t. 107, 2000, pp. 23-40. 4(26) «Hommages et supplications au tombeau de saint Yves.» 5(27) ...Le reste est indéchiffrable ${ }^{7}$.

Ce portail Saint-Yves se singularise des autres portails par un décor plus abondant et plus riche; il possède trois voussures sculptées (c'est-à-dire autant que le portail central) contre deux pour les autres entrées et son revers se signale par la présence d'un liseré feuillagé sculpté autour du tympan. Ce liseré court à la base de la baie formée par ce tympan et disparaît à l'ouest et à l'est dans le mur du frontispice ou dans les retombées des premières grands arcs de la nef. Ces dispositions qui contribuent à isoler cette entrée des autres portes de l'édifice doivent être mises en relation avec l'implantation même de ce portail dans la façade et son inscription dans la ville.

Le portail saint Yves en effet est directement rattaché au château des ducs de Bretagne au sud de la cathédrale auquel il conduit par la rue Mathurin Rodier ${ }^{8}$. Le tracé actuel de la rue Mathurin Rodier est issu de l'extension sur la rue des bâtiments longeant le flanc sud de l'édifice, et cela à l'époque moderne. Initialement cependant, les plans conservés aux archives municipales le montrent ${ }^{9}$, cette rue conduisait plus directement encore du château à la porte Saint-Yves qui se trouvait alors exactement dans son axe, marquant ainsi combien ce portail était lié au pouvoir temporel. Les sources insistent sur le statut de cette entrée de l'édifice. C'est en effet par le terme d'«entrée du château » ou, plus explicite encore, d' "Introïtu Regis » que certains documents le désignent ${ }^{10}$. Selon toute vraisemblance, c'est à cette fonction d'entrée du duc qu'il faut rapporter la présence d'un balcon à droite du tympan. Ce

${ }^{7}$ L'examen direct des groupes altérés de la demi voussure extérieure est, à la faveur d'échafaudages de restauration, permettra peut-être à l'avenir de compléter cette description.

${ }^{8}$ Château qui n'avait pas, lors de la construction et la décoration des portails de la cathédrale, le visage actuel, pour une très large part issu des reconstructions de François II et Anne de Bretagne (LA BORDERIE (Arthur Le Moyne de), «Mandements de François II pour la reconstruction du château de Nantes », dans Bulletin de l'Association Bretonne, 1893 ; LA BORDERIE (Arthur Le Moyne de), « Mathurin Rodier, architecte du château et de la cathédrale de Nantes », dans Revue des Provinces de l'Ouest. Bretagne et Poitou, Paris, 1854, pp. 598-599).

${ }^{9}$ Archives municipales de Nantes, II $160 * \mathrm{n}^{\circ} 10$ «Plan partiel des cours, place d'armes et Saint-Pierre », anonyme, XVIIIe siècle. Voir également «Plan de Nantes avec les changements et augmentations qu'on y a fait depuis 1757, chez Le Rouge », mise à jour du plan Cacault, 1766 (Nantes, musée Dobrée)

${ }^{10}$ Arch. dép. Loire-Atlantique, 107 J 251, «Papiers Bourdeaut». Décisions capitulaires, 28 mai 1518 («Dni concluserunt quod misiator operis faciat amovere terras in cimeterio et in introitu Regis ponantur gentes ad custodiendas imagines portalis ne dirrumpantur $\gg)$. 
Jean-Marie Guillouët, «L'iconographie de saint Yves et la politique dynastique des ducs Montfort à la fin du

Moyen Âge » ("Saint Yves' Iconografy and Dynastic Strategy in Late Medieval Britany »), in Annales de Bretagne et des Pays de l'Ouest, t. 107, 2000, pp. 23-40. balcon est d'ailleurs desservi par un escalier dont la porte d'accès dans le bas-côté de l'église était initialement surmontée des armes de de Bretagne ${ }^{11}$.

Une copie du livre des anniversaires de la cathédrale de Nantes, conservée aux archives départementales de Loire-Atlantique, permet de comprendre l'organisation générale de cette façade et l'implantation du portail Saint-Yves. Trois axes partagent les dévotions des fondateurs nantais à la fin du Moyen Âge. C'est, bien sûr, en premier lieu vers les grandes figures de la chrétienté latine que se tournent les auteurs des services (saint Gabriel Archange, saints Cosme et Damien, saint Georges, saint André, saint Paul, saint Etienne, saint Nicolas..., et bien entendu les saints Pierre et Paul). Aucune de ces dévotions n'est atypique dans le contexte français de la fin du Moyen Age ; certaines d'entre elles, comme le culte de saint Christophe, sont même extrèmement répandues et populaires. Les figures du christianisme breton apparaissent ensuite en nombre un peu inférieur et marquent l'appartenance au duché (saint Guillaume évêque de Saint-Brieuc, saint Fiacre, saint Yves, saint Paul de Léon, saint Brieuc...). Enfin, l'Eglise nantaise fournit le dernier contingent d'intercesseurs vers lesquels se tournent les fidèles (saint Félix, saint Clair, saint Gohard, saint Martin de Vertou, saint Similien, saints Donatien et Rogatien) ${ }^{12}$.

Cette dialectique de l'universel chrétien, du régional et du local dans les dévotions nantaises à la fin du Moyen Âge évoque les dispositions mêmes de la façade nantaise. A l'ouest, à l'endroit le plus éminemment symbolique, se trouvent les portails Saint-Pierre et Saint-Paul alors que les dévotions régionales et locales sont repoussées au sud et au nord du massif de façade, respectivement dans les portails Saint-Yves et Saints-Donatien et Rogatien. Les dispositions mêmes de l'architecture semblent relayer cette interprétation. C'est en effet au sud et au nord que sont repoussées les influences décoratives bretonnes comme les troncs écôtés qui décorent les ébrasements des portails latéraux dont les origines sont à rechercher du côté de l'architecture cornouaillaise contemporaine.

11 Nantes. La cathédrale, Inventaire général des monuments et des richessse artistiques de la France, « Images du patrimoine », Nantes, 1991, p. 28.

12 Pour ce paragraphe, nous reprenons notre article : GUILLOUËT (Jean-Marie), « Un témoin des dévotions nantaises à la fin du Moyen Âge », dans Bulletin de la société archéologique et historique de Nantes et de LoireAtlantique, t. 133, 1998, pp. 83-95. 
Jean-Marie Guillouët, «L'iconographie de saint Yves et la politique dynastique des ducs Montfort à la fin du Moyen Âge » ("Saint Yves' Iconografy and Dynastic Strategy in Late Medieval Britany »), in Annales de Bretagne et des Pays de l'Ouest, t. 107, 2000, pp. 23-40.

Comment la figure de saint Yves, au point d'articulation de ces deux préoccupations (le pouvoir et ses représentations d'un côté ${ }^{13}$, l'identité culturelle bretonne de l'autre), doit-elle être interprétée? A travers les sources de la fin du Moyen Âge, celle-ci semble devoir se présenter sous des dehors qui ne permettent pas d'établir sans discussion l'équation : saint Yves égal Bretagne. Figure tutélaire d'une "bretonnité" revendiquée par le pouvoir ducal, saint Yves endosserait dans ce cas un rôle qui n'est malheureusement, et cela en dépit du caractère séduisant de cette interprétation, jamais attesté aussi clairement dans les sources. L'image de saint Yves à la fin du Moyen Âge semble en effet être prise dans un réseau d'enjeux politiques, culturels et religieux qui dépassent la simple affirmation de l'identité bretonne.

\section{Les chroniqueurs, saint Yves et l'identité nationale}

Pour interroger la dimension politique de saint Yves à la fin du Moyen Âge, un témoignage semble s'imposer en premier lieu, celui de la chronique bretonne. Dans l'entourage direct du pouvoir, les chroniqueurs bretons des XIVe et XVe siècles montrent un attachement profond à l'identité politique et culturelle de la Bretagne face au royaume de France. Le contexte politique particulièrement tendu entre le duc et son suzerain (en dépit de périodes de détente, voire d'entente) tout au long du XVe siècle et les difficultés de la couronne ducale à partir des années 1460 contribuent à expliquer le caractère militant de cette chronique qui mérite bien le titre de «sous-produit de la politique ${ }^{14}$ ». La défense des prérogatives et privilèges du duché constitue un des enjeux majeurs de l'historiographie bretonne de la fin du Moyen Âge ${ }^{15}$; à ce

\footnotetext{
${ }^{13}$ La question du rôle des ducs de Bretagne dans la construction de la cathédrale de Nantes ne saurait être évoquée en quelques lignes. Rappelons seulement, pour aller dans le sens de l'analyse et pour se limiter à des données certaines, l'importance financière et politique de Jean V et de ses successeurs dans le chantier de SaintPierre. Celui-ci, à bien des égards, peut être considéré comme un « chantier ducal » (sur ces questions, nous nous permettons de renvoyer à notre mémoire de maîtrise, La façade occidentale de la cathédrale Saint-Pierre et Saint-Paul de Nantes. Etude architecturale, Paris-IV, sous la direction de Anne Prache et Dany Sandron, 1995).

${ }^{14}$ B. GUENÉE cité par KERHERVÉ (Jean), « Aux origines d'un sentiment national. Les chroniqueurs bretons de la fin du Moyen Âge », dans Bulletin de la société archéologique du Finistère, 1980, 108, pp. 165-206 ; p. 195.

${ }^{15}$ Cette « défense de l'identité » ne doit pas laisser penser qu'il s'agit de libelles au service d'une propagande grossière ; Dominique Philippe a montré comment se construit, à la fin du Moyen Âge, une véritable méthode historique chez Pierre Le Baud par exemple (PhILIPPE (Dominique), « L'élaboration d'une méthode historique : la chronique bretonne aux XIVe et XVe siècle », dans Annales de Bretagne, 1997, 104, n², pp. 47-58).
} 
Jean-Marie Guillouët, « L’iconographie de saint Yves et la politique dynastique des ducs Montfort à la fin du Moyen Âge » (« Saint Yves' Iconografy and Dynastic Strategy in Late Medieval Britany »), in Annales de Bretagne et des Pays de l'Ouest, t. 107, 2000, pp. 23-40. titre, elle prend une part importante dans la constitution d'un « sentiment national » breton ${ }^{16}$. Or, au sein de ce corpus de textes, la figure de saint Yves se remarque surtout pas son relatif effacement. Comme l'a relevé Dominique Philippe, les mentions de saint Yves dans la chronique bretonne de la fin du Moyen Âge sont finalement relativement peu nombreuses ${ }^{17}$. C'est Alain Bouchart qui lui consacre le plus d'attention avec plus de trois feuillets de sa chronique $^{18}$ contre seulement quinze lignes dans la première histoire de Bretagne de Pierre le Baud et onze dans la seconde ${ }^{19}$. Le même Pierre le Baud ignore d'ailleurs saint Yves dans sa Compillation des croniques et ystoires des Bretons de $1480^{20}$. Jean de Saint Paul ou l'Anonyme de la chronique de Saint-Brieuc (pourtant « un fougueux patriote breton, chez qui l'amour du pays s'accompagnait d'un violent sentiment xénophobe à l'égard des Anglais et des Français $\left.{ }^{21} »\right)$ ne le mentionnent pas plus ; tout comme Guillaume de Saint André ${ }^{22}$ ou,

${ }^{16}$ Kerhervé (Jean), « Aux origines d'un sentiment national. Les chroniqueurs bretons de la fin du Moyen Âge », dans Bulletin de la société archéologique du Finistère, 1980, 108, pp. 165-206.

${ }^{17}$ PhILIPPE (Dominique), L'histoire en Bretagne du XIVe au XVIe siècle ou la défense de l'identité, Thèse dactylographiée, Brest, 1988, p. 214.

18 Alain Bouchart, Grandes chroniques de Bretagne, texte établi par Marie-Louise AugER, Gustave JEANNEAu et Bernard GuenÉE (dir.), CNRS, Paris 1986, t. II, pp. 14-21.

19 « En l'an 1303, le dix-neufiesme iour du mois de May à un Dimanche, trespassa de ce siecle Maistre Yves Harlory venerable prestre du Diocese de Trecorense, homme efficacement instruit en Droict canon \& science divine, \& fut inhumé en l'Eglise cathédral de l'Antreguer. Lequel Yves, combien qu'il fust de Tres-nobles parens, se fist advocat des poures \& miserables personnes, reduisant de toutes ses forces les litigans à paix \& concorde. Et fut de sit très-grande humilité merveilleuse, saincteté \& et incrédible austérité de vie, resplendissant avant sa mort \& après tant d'innumerables, merveilleux, \& evidens miracles, qu'il n'est point leu par autre confesseur tant en avoir esté fait, pour le mérite desquels long-temps après pape Clement sixiesme l'escripsit solemnellement ou cathologue des saincts confesseurs. » (Histoire de Bretagne avec les chroniques des maisons de Vitré et de Laval par Pierre le Baud, chantre et chanoine de l'église collégiale de Nostre-Dame de Laval, Trésorier de la Madeleine de Vitré, conseiller et ausmonier d'Anne de Bretagne, reine de France, Paris, Gervais Alliot, 1638, p. 254).

${ }^{20}$ Bibliothèque nationale, ms.fr. 8266 .

${ }^{21}$ KeRHERVÉ (Jean), « Aux origines d'un sentiment national..., p. 205.

22 Jean de SAInt Paul, Chroniques de Bretagne, publié par Arthur de LA BorderIE, Nantes, Société des Bibliophiles Bretons, 1881 ; Chronicon Briocense, dans MORICE (Dom Pierre Hyacinthe), Mémoire pour servir à l'histoire ecclesiastique et civile de la Bretagne, Tiré des archives de cette province, de celles de France et d'Angleterre, des receuils de plusieurs sçavants antiquaires et mis en ordre par Dom Pierre Hyacinthe Morice, 3 vol. , 1742-1746, t. I, col. 7 à 102 (l'édition proposée par Lobineau au début du XVIIIe siècle est en grande partie tronquée) ; et Guillaume de SAINT ANDRÉ, C'est le livre du bon Jehan, duc de Bretagne [à la suite de CUVELIER, Chronique de Bertrand du Guesclin], Paris, Charrière, 1839, t. II, pp. 421-560. 
Jean-Marie Guillouët, «L'iconographie de saint Yves et la politique dynastique des ducs Montfort à la fin du Moyen Âge » ("Saint Yves' Iconografy and Dynastic Strategy in Late Medieval Britany »), in Annales de Bretagne et des Pays de l'Ouest, t. 107, 2000, pp. 23-40. plus tard, Bertrand d'Argentré ${ }^{23}$. Il est pourtant permis de croire que les auteurs convoqués n'auraient certainement pas raté un aussi bel argument à leur démonstration. Même Alain Bouchart, le plus prolixe des chroniqueurs au sujet de saint Yves (qui doit peut-être cet attachement à sa formation de juriste) semble admirer en premier lieu l'ascétisme chrétien de 1'official de Tréguier, sans insister sur son caractère "national"24.

C'est bien plus tard semble-t-il, uniquement au XVIe ou au XVIIe siècle, qu'il faut situer l'accession d'Yves de Kermartin au rang de saint «authentiquement breton ${ }^{25}$ ». A la différence des chroniqueurs médiévaux, Albert le Grand ou Lobineau sont tout à fait explicites sur la dimension bretonne du personnage. Il est, pour le dominicain, « le patron universel de la Bretagne armorique mais spécialement de l'évêché de Tréguier $^{26}$ ». Lobineau avance de manière beaucoup plus tournée ses arguments et explique que la détermination du pape Clément VI à canoniser le saint reposait entre autres sur le fait que, comme lui, «il devoit aussi être censé Breton (lui Clément [VI]) puisqu'il étoit du païs de Limoges qui étoit tombé par alliance sous la domination des ducs de Bretagne $^{27} \gg$. Il est donc clairement établi, au début du XVIIIe siècle, que saint Yves est, avant tout, un saint breton; au point qu'une solidarité régionale, même lointaine, puisse être évoquée pour expliquer son accession aux autels. Mais aux XIVe et XVe siècles la figure du recteur de Trédrez ne semble pas devoir être limitée à l'identité bretonne, presque entièrement passée sous silence par la chronique contemporaine.

Il convient de chercher ailleurs l'explication de la présence de saint Yves dans les voussures nantaises. Un détour par l'histoire troublée du duché de Bretagne au XIVe siècle et les aléas de la canonisation du saint de Kermartin en fournit les clefs.

${ }^{23}$ PhILIPPE (Dominique), L'histoire en Bretagne du XIVe au XVIe siècle..., p. 214.

${ }^{24}$ PhILIPPE (Dominique), L'histoire en Bretagne du XIVe au XVIe siècle..., p. 214. Alain Bouchart était semble-t-il « fort préoccupé de l'histoire de saint Yves » (LA BORDERIE (A. de), Les monuments originaux de l'histoire de saint Yves. Rapport à Mgr. l'évêque de Saint-Brieuc et Tréguier, Saint-Brieuc, 1885, p. 14).

${ }^{25}$ Par prescrit du pape Pie XI, le 12 mars 1924, saint Yves deviendra finalement co-patron de la Bretagne avec sainte Anne (Debidour (V. H.), «Saint Yves, étude iconographique », dans Mémoires de la société d'histoire et d'archéologie de Bretagne, 1949, 29, pp. 35-44 ; p. 36).

${ }^{26}$ LE GRAND (Albert), La vie, gestes, morts et miracles des saints de la Bretagne armoricaine, Rennes, 1659, p. 156.

${ }^{27}$ LoBINEAU (Dom Guy Alexis), La vie des saints de Bretagne et des personnes d'une éminente piété qui ont vécu dans la même province, Rennes, 1724. 
Jean-Marie Guillouët, «L'iconographie de saint Yves et la politique dynastique des ducs Montfort à la fin du Moyen Âge » (« Saint Yves' Iconografy and Dynastic Strategy in Late Medieval Britany »), in Annales de Bretagne et des Pays de l'Ouest, t. 107, 2000, pp. 23-40.

Saint Yves, Charles de Blois et la guerre de succession de Bretagne

La dévolution du trône de Bretagne après la mort de Jean III, le 30 avril 1341, a constitué une des modalités de l'affrontement général entre la France et l'Angleterre. Issu du mariage d'Arthur II avec Marie de Ségur-Comborn, vicomtesse de Limoges, Jean III meurt sans enfants et sans avoir pris la précaution de régler sa succession ${ }^{28}$. Son frère Guy de Penthièvre était mort en 1331 mais avait eu une fille de Jeanne d'Avaugour, Jeanne de Penthièvre. En 1337, cette dernière épouse Charles de Blois, fils du comte de Blois et de Marguerite, la sœur de Philippe VI. C'est par conséquent au titre de sa femme que Charles de Blois, neveu du roi de France, prétend au trône ; mais il s'oppose alors à Jean de Montfort, demi-frère du défunt duc, issu du second mariage d'Arthur II avec Yolande de Dreux, comtesse de Montfort. Bien qu'il meure assez rapidement en 1345, Jean de Montfort laissera de son mariage avec Jeanne de Flandre un fils qui, vainqueur finalement du parti Penthièvre, règnera sous le nom de Jean IV. A travers la lutte que se livreront Charles de Blois et Jean de Montfort (puis son fils) se cristalisent les enjeux de la politique internationale du moment : Charles de Blois, un prince étranger au duché et allié à la maison de France d'un côté ; Jean de Montfort, breton lui, mais soutenu par ses alliés anglais, de l'autre.

La complexité apparente de la situation peut se résumer de manière assez paradoxale: alors que la première phase de la guerre de Cent Ans et les crises successorales en France poussent les jurisconsultes du royaume à formuler l'exclusion des femmes de la dévolution de la couronne au profit du plus proche parent par les hommes ${ }^{29}$, ces mêmes jurisconsultes soutiennent en Bretagne les droits de Charles de Blois, pourtant prétendant au trône au titre de sa femme, nièce du duc défunt. A l'inverse, alors que le roi d'Angleterre prétend pouvoir hériter du trône de France par le biais d'Isabelle, il défend en Bretagne les prétentions de Jean de Montfort qui se fondent précisément sur l'exclusion des femmes. Ce jeu croisé d'arguments et de raisons politiques se complique encore chez Montfort par l'évocation d'arguments juridiques lourds mais dangereux. Les partisans de Jean de Montfort rappellant

\footnotetext{
${ }^{28}$ Il semble avoir eu du mal à arbitrer entre ses proches et, à l'extrémité de sa vie, avait demandé à ce sujet « que por Dieu on le laissast en paix, qu'il ne vouloit pas charger son âme » (LEGUAY (Jean-Pierre), MARTIN (Hervé), Fastes et malheurs de la Bretagne ducale 1213-1532, Rennes Ouest-France, 1982, p. 100).

${ }^{29}$ Le choix de 1328 , celui d'un roi français par les barons, qui a ôté à Isabelle tout rôle dans la succession royale, inaugure alors l'impossibilité signifiée aux femmes de faire «le pont et la planche », c'est-à-dire de transmettre un droit qu'elles ne peuvent exercer (sur ces questions voir ViollET (Paul), « Comment les femmes
} 
Jean-Marie Guillouët, «L'iconographie de saint Yves et la politique dynastique des ducs Montfort à la fin du

Moyen Âge » (« Saint Yves' Iconografy and Dynastic Strategy in Late Medieval Britany »), in Annales de Bretagne et des Pays de l'Ouest, t. 107, 2000, pp. 23-40.

que la Bretagne est devenue depuis 1297 un duché-pairie et qu'à ce titre, «membre et partie de la couronne ${ }^{30} »$, elle doit appliquer la législation en vigueur dans le royaume !

Bien que saint Yves, mort en réputation de sainteté à l'aube du 19 mai 1303, appartienne biographiquement au XIIIe siècle ; sa légende ne peut se détacher du contexte qui vient d'être évoqué. Politiquement, saint Yves appartient au XIVe siècle. C'est en effet au moment des premiers combats de la guerre de succession de Bretagne que la canonisation de l'official de Tréguier prend place. Jean III le premier avait adressé une supplique à Clément V, moins de dix ans après la mort du recteur de Trédrez, afin de demander l'ouverture d'une enquête canonique. La disparition du pape en 1314 puis l'interrègne qui suit obligent le duc à renouveler sa demande auprès du pape Jean XXII ${ }^{31}$. Pour cette seconde demande, il s'assure (et le fait est notable) le soutien et l'appui du roi et de la reine de France, de l'université de Paris et de nombreux évêques ou archevêques français. La curie s'opposant dans un premier temps à l'enquête, la situation ne se débloquera qu'avec le voyage à Avignon, en décembre 1329, de Guy de Bretagne, comte de Penthièvre, et de l'évêque de Tréguier, Yves de Boisboissel, muni d'une procuration de son chapitre ${ }^{32}$. Le 26 février 1330 en effet, une bulle de Jean XXII permettant l'ouverture d'un procès répond enfin à la demande du duc de Bretagne $^{33}$. L'enquête in partibus effectuée à Tréguier du 23 juin au 4 août 1330 par les commissaires apostoliques est finalement présentée en plein consistoire le 4 juin $1331^{34}$. Cependant, la mort de Jean XXII en 1334 bloque à nouveau la procédure qui s'enlise. Cette situation perdure d'autant plus longtemps que le successeur de Jean XXII, Benoît XII, ne montre aucun zèle. En février 1345, alors que la procédure canonique est au point mort, c'est Charles de Blois qui obtient la réunion d'un consistoire à Avignon pour faire entendre deux

ont été exclues de la succession à la couronne », dans Mémoires de l'Académie des Inscriptions et Belles Lettres, XXXIV (2), 1895, pp. 125-178).

${ }^{30}$ LeGUAY (Jean-Pierre), MARTIN (Hervé), Fastes et malheurs..., p. 99.

${ }^{31}$ La Borderie (A. de), Daniel (abbé J.), Perquis (R. P.), Tempier (D.), Monuments originaux de l'histoire de saint Yves publiés pour la première fois, Saint-Brieuc, Prud'Homme, 1887, p. XXVII. Un résumé clair mais sans référence des étapes de la canonisation de saint Yves peut être trouvé dans CASSARD (JeanChristophe), Saint Yves de Tréguier. Un saint du XIIIe siècle, Paris, Beauchène, 1992, pp. 129-139 ( «L'accession de saint Yves à la gloire des autels »).

\footnotetext{
${ }^{32}$ CASSARD (Jean-Christophe), Saint Yves de Tréguier..., p. 132.

${ }^{33}$ LA BORDERIE (A. de), Les monuments originaux de l'histoire de saint Yves. Rapport à Mgr...., p. 4.

${ }^{34}$ Ibidem.
} 
Jean-Marie Guillouët, «L'iconographie de saint Yves et la politique dynastique des ducs Montfort à la fin du Moyen Âge » ("Saint Yves' Iconografy and Dynastic Strategy in Late Medieval Britany »), in Annales de Bretagne et des Pays de l'Ouest, t. 107, 2000, pp. 23-40. nouveaux miracles récents imputables au saint ${ }^{35}$. A cette occasion, il demande et obtient la réouverture du procès de canonisation pour le financement duquel il versera jusqu'à 3000 florins $^{36}$. La bulle de canonisation finalement délivrée par Clément VI, le 19 mai 1347 rappelle d'ailleurs le rôle capital joué par Charles de Blois dans l'aboutissement de la procédure $^{37}$.

A travers cet aperçu historique du procès de canonisation de saint Yves, l'importance de l'action de Charles de Blois pour la réussite de la procédure concernant l'official de Tréguier apparaît pleinement. Son engagement dans ce combat est souligné par tous ses contemporains qui relèvent régulièrement l'énormité de la somme par lui versée. Bien que l'initiative, c'est à dire la demande d'enquête canonique, ait été introduite par Jean III très vite après la mort du saint puis soutenue par Guy de Bretagne, duc de Penthièvre, le neveu du roi de France est apparu comme le principal responsable de l'accession de saint Yves aux autels. Charles de Blois exploite ainsi un sentiment national breton afin de s'assurer le soutien, ou pour le moins la neutralité, d'une partie de la population du duché. Saint Yves a donc été, dans l'esprit de Charles de Blois, un saint offrant une "bonne prise" à l'identité bretonne et le caractère politique de cette dévotion a été très largement souligné par de nombreuses études ${ }^{38}$. Celle-ci fut relayée par des cultes encore plus spécifiquement politiques comme celui des saints rois bretons du Haut Moyen Age auquel trois témoins du procès de canonisation de Charles de Blois font mention. La symbolique de cette dévotion aux anciens rois de Bretagne apparaît

${ }^{35}$ Il s'agit de deux sauvegardes de naufrage (cf. PLAINE (Dom François), Histoire du bienheureux Charles de Blois ; à la suite des Monuments du procès de canonisation du bienheureux Charles de Blois, duc de Bretagne 1320-1364, Saint-Brieuc, Prud-Homme, 1921, p. 547). Yves n'avait - bien entendu - pas attendu le jugement de canonisation pour opérer de nombreux miracles à son tombeau ou à distance.

${ }^{36}$ CASSARD (Jean-Christophe), Saint Yves de Tréguier..., p. 134. De nombreux témoins du procès de canonisation de Charles de Blois attestent cette libéralité, par exemple le clerc Guillaume André (Monuments du procès de canonisation du Bienheureux Charles de Blois..., p. 93).

${ }^{37}$ «Dux Britanniae, qui nunc est, ad nos in propria personna venit, et in sui praesentari egotium etiam proponi fecit in consistorio, et ipsemet ibidem duo miracula retulit », Boll. Maii, IV, p. 579, A et B cité par LA Borderie (A. de), DANiel (abbé J.), Perquis (R. P.), TeMPier (D.), Monuments originaux de l'histoire de saint Yves ..., p. XLIX. Charles de Blois est ici mentionné comme « duc de Bretagne ».

${ }^{38}$ On consultera sur la période les études essentielles de Michael Jones, réunies dans JONES (Michael), The Creation of Britany, a Late Medieval State, Londres, 1988. 
Jean-Marie Guillouët, «L'iconographie de saint Yves et la politique dynastique des ducs Montfort à la fin du Moyen Âge » ("Saint Yves' Iconografy and Dynastic Strategy in Late Medieval Britany »), in Annales de Bretagne et des Pays de l'Ouest, t. 107, 2000, pp. 23-40. clairement $^{39}$. A la cathédrale de Rennes (église du couronnement), une chapelle avait été financée par Charles de Blois en l'honneur des saints Judicaël et Salomon, dédicace à laquelle se trouvaient significativement associés saint Yves et les saints Donatien et Rogatien ${ }^{40}$ !

La réussite de cette entreprise d'appropriation conduite par Charles de Blois n'est plus remise en doute. Pour Jean-Yves Copy, Charles de Blois « devient breton à part entière ${ }^{41} »$. Lors de son procès de canonisation tenu à Angers en 1371, au moins deux témoins y jugent important de rappeler que Charles de Blois leur était compatriote, eux-mêmes étant bretons ${ }^{42}$. L'association saint Yves/Charles de Blois apparaît sous la plume de Guillaume de la Pérenne qui raconte comment les troupes bretonnes qui se portaient au service d'Urbain V en 1368 invoquaient ensemble les deux personnages, alors indirectement désignés comme bretons :

« Les Bretons crient: Vive l'Eglise

En appelant tous à voix vive

Voustre merci, Charles et Yves

Ce sont deus seins du paradis,

Qu'aux dits Bretons furent amis ${ }^{43} »$.

${ }^{39}$ VAUCHEZ (André), « Le duc Charles de Blois ( $†$ 1364) et le culte des saints rois bretons du Haut Moyen Age », dans Moyen Age. Culture, éducation et société. Etudes offertes à Pierre Riché, La Garenne-Colombe, 1990, pp. 605-615.

${ }^{40}$ «Item fecit fieri in dicta ecclesia Redonensi unam capellam in honorem sanctorum Salomonis martiris et Judicaelis confessori, quondam regum Brittanie, ac sanctorum martyrum Donaciani et Rogatiani et sancti Yvonis confessoris » (chevalier Thibault de Belozac, de Noyal-sur-Seiche (Ile-et-Vilaine), dans Monuments du procès de canonisation du bienheureux Charles de Blois..., p. 68 ; cité dans VAuchez (André) ; «Le duc Charles de Blois et le culte des saints rois bretons... », p. 606).

${ }^{41}$ Copy (Jean-Yves), Art, société et politique au temps des ducs de Bretagne. Les gisants haut-bretons, Paris, 1986, p. 112.

${ }^{42}$ Monuments du procès de canonisation du bienheureux Charles de Blois..., p. 247 (Yves de Alnete, de parrochia Nova Leonensis Diocesis) ; voir également p. 340.

${ }^{43}$ Guillaume de la PÉRenne, Geste des Bretons en Italie, édition dans Morice, Preuves..., t. II, col. 134172 ; col. 148. André Vauchez omet de citer le troisième vers de cet extrait qui pourtant est important dans le contexte étudié ici puisqu'il semble attester que cette association Charles de Blois/saint Yves est issue d'un contexte blésois. Charles de Blois en effet, à la canonisation pourtant problèmatique, est mentionné comme « sein[s] du paradis »; or ce culte à « saint » Charles de Blois est directement lié à la volonté politique du parti Penthièvre et fut, à ce titre, largement combattu par les Montfort (HERY (Laurent), «Le culte de Charles de Blois : résistances et réticences » dans Annales de Bretagne et des Pays de l’Ouest, 1996, t. 103, nº 2, pp. 39-56). 
Jean-Marie Guillouët, «L'iconographie de saint Yves et la politique dynastique des ducs Montfort à la fin du Moyen Âge » ("Saint Yves' Iconografy and Dynastic Strategy in Late Medieval Britany »), in Annales de Bretagne et des Pays de l'Ouest, t. 107, 2000, pp. 23-40.

Cette association saint Yves/Charles de Blois est attestée dans des contextes essentiellement francophiles, plutôt favorables au parti Penthièvre et trahit bien l'orientation politique de cette dévotion. Au moment de sa mort, Bertrand du Guesclin recommande son âme à Charles de Blois qu'il considère comme saint; de manière symptomatique, il ordonne alors «qu'un pelerin pour nous soit enveé en veage pour nous a St Charles et a St Yves en Bretagne » et qu'il soit porteur de mille livres de cire ${ }^{44}$. De la même façon, parmi les dépositions des témoins du procès de canonisation de Charles de Blois à Angers, qui ont donc bravé l'interdiction ducale et les chicanes de ses agents, on relève à de nombreuses reprises ${ }^{45}$ cette association par le biais d'un rappel des pèlerinages du duc défunt à Tréguier ou de sa dévotion particulière à saint $\mathrm{Yves}^{46}$.

\section{La récupération par les Montfort et la façade nantaise}

Cette orientation politique de l'iconographie de saint Yves est a priori contradictoire avec la présence d'un cycle de saint Yves dans les voussures nantaises. Le règne de Jean V

${ }^{44}$ Morice, Preuves..., t. II, col. 287.

${ }^{45}$ Monuments du procès de canonisation du bienheureux Charles de Blois, duc de Bretagne 1320-1364, Saint-Brieuc, Prud'Homme, 1921. On notera les témoignages suivants : Olivier de Buyguonig, paroisse de SaintTudual au diocèse de Dôle (p. 32) ; maître Rollandus de Coestelles, paroisse de Mautref (p. 45); frère Paganus de Kelen, du couvent franciscain de Guimgamp au diocèse de Tréguier (p. 51) ; frère Derianus Parvi, du même couvent (p. 55); Theobaldus de Belozac, paroisse de Noyalo sur Siccam au diocèse de Rennes (p. 68) ; Pietrus de Cathedra, archidiacre et chanoine de Tréguier (p. 75) ; Colinus Audigerii, paroisse de Ploefragan au diocèse de Saint-Brieuc (p. 82) ; Alanus Radulphi (p. 87) ; Yvo Crenan, paroisse de Plaesidii au diocèse de Tréguier (p. 91); Guillaume André de Meduane au diocèse du Mans (p. 95); Gauffridus Rabin, dominicain de Nantes (p. 102) ; Jeaen Galvani, au diocèse de Saint-Malo (p. 105) ; Gauffridus de Chesnelli (p. 127) ; Olivierus Theobaldi (p. 135); Micheletus Barbeloti (p. 164); Johannes Forestarii (p. 178); Prigencio Hammonou (p. 185); Yvo Beguti (p. 323); Guillermus Anseau (p. 343); Hamo de Langouez (p. 354); Johannes Hamonou (p. 382); Alanus Gyiou (p. 393).

${ }^{46}$ Une telle association est finalement assez compréhensible. Charles de Blois fut un grand zélateur de l'official de Tréguier et le premier propagateur de son culte. La profondeur de l'attachement de Charles de Blois pour saint Yves apparaît dans de nombreux témoignages. Ce qui rend la réalité de cette dévotion tout à fait crédible, c'est le fait que certains d'entre eux ne parviennent pas à faire taire complètement les « murmures de désapprobation » que suscite son excessive ascèse, inhabituelle chez un laïc (VAUCHEZ (André), La sainteté en Occident aux derniers siècles du Moyen Age d'après les procès de canonisation et les documents hagiographiques, BEFAR, Paris, De Boccard, 1998, p. 424). Derrière les témoignages les plus favorables au duc, lors même de son procès en canonisation, sourd ce reproche qui trahit alors certainement une réelle et profonde piété. Qu'elle fût sincère, n'empêche pourtant pas cette dévotion d'avoir été essentiellement politique. 
Jean-Marie Guillouët, «L'iconographie de saint Yves et la politique dynastique des ducs Montfort à la fin du Moyen Âge » ("Saint Yves' Iconografy and Dynastic Strategy in Late Medieval Britany »), in Annales de Bretagne et des Pays de l'Ouest, t. 107, 2000, pp. 23-40. inaugurera cependant au début du XVe siècle une stratégie systématique de récupération (Jean-Yves Copy parle de « $\left.\operatorname{captation}^{47} »\right)$ par les Montfort de l'imagerie introduite par Charles de Blois dans le duché ${ }^{48}$. A ce titre, et bien que le sentiment national breton soit antérieur à la crise successorale du milieu du XIVe siècle ${ }^{49}$, on voit combien cette dernière a profondément influencé la conception du pouvoir dynastique en Bretagne et ses représentations. Charles de Blois est généralement crédité de l'introduction en Bretagne, dans les sceaux et les monnaies, de la représentation du prince en majesté, dérivée de l'imagerie royale, ainsi que des attributs du pouvoir habituellement réservés au roi français comme la titulature «Par la grâce de Dieu ${ }^{50}$ ». Cette titulature, comme l'utilisation de la couronne fleuronnée à la place du cercle ducal (lequel est alors porté par le fils aîné du duc ${ }^{51}$ ), seront largement reprises par les successeurs de Jean de Montfort et serviront ultérieurement à asseoir leurs prétentions face au royaume de France durant toute la seconde moitié du XVe siècle. Pour Jean-Christophe Cassard le trône ducal bénéficie, à l'occasion des débats de la guerre de succession, d'apports contribuant à dissiper « certaines inhibitions repérables depuis le commencement du XIVe siècle ${ }^{52} »$. Grâce à l'action de Charles de Blois, le trône dont hérite Jean $\mathrm{V}$ de Montfort sort des querelles du XIVe siècle breton enrichi dans les instruments symboliques de sa domination et de son affirmation.

${ }^{47}$ COPY (Jean-Yves), Art, société et politique..., p. 116.

${ }^{48} \mathrm{Ce}$ trait d'intelligence politique suffirait à rendre un peu relief à la figure de Jean $\mathrm{V}$, objet d'appréciations contradictoires et longtemps taxé de faiblesse, de médiocrité (sur ces questions, confronter les articles de BourdeAut (abbé A.), «Etude sur le caractère moral de Jean V, duc de Bretagne », dans Bulletin de la Société Archéologique et Historique de Nantes et de la Loire Inférieure, 1916 ; et KNOWLSON (G. A.), Jean V, duc de Bretagne, et l'Angleterre (1399-1442), Cambridge-Rennes, 1964).

49 JONES (Michaël), " "Mon Païs et ma nation" : Breton Identity in the Fourtheenth Centruy », dans The Creation of Brittany..., pp. 283-307; p. 290.

${ }^{50}$ CARON (Marie-Thérèse), Noblesse et pouvoir royal en France XIIIe-XVIe siècle, Paris, Armand-Colin, 1994 , p. 102 et MÉRINDOL (Christian de), «L'imaginaire du pouvoir à la fin du Moyen Âge. Les prétentions royales", dans Représentation, pouvoir et royauté à la fin du Moyen Âge (actes du colloque organisé par l’Université du Maine, les 25 et 26 mars 1994), Paris, Picard, 1995, pp. 65-92 ; p. 82.

51 Pocquet DU Haut-Jussé (B.-A.), «Couronne fermée et cercle ducal en Bretagne », dans Bulletin philologique et historique (jusqu'à 1715) du comité des travaux historiques et scientifiques, années 1951/1952, Paris, PUF, 1953, pp. 103-112 reste encore une base indispensable de travail sur la question.

52 CASSARD (Jean-Christophe), « La tradition royale en Bretagne Armorique », dans Revue Historique, 569, janv.-mars 1989, pp. 15-45; p. 45. 
Jean-Marie Guillouët, «L'iconographie de saint Yves et la politique dynastique des ducs Montfort à la fin du Moyen Âge » ("Saint Yves' Iconografy and Dynastic Strategy in Late Medieval Britany »), in Annales de Bretagne et des Pays de l'Ouest, t. 107, 2000, pp. 23-40.

C'est dans ce contexte que l'iconographie de saint Yves prend toute sa complexité. Si l'appropriation par Charles de Blois de saint Yves, un saint « breton, connu pour son intégrité et son sens de la justice ${ }^{53}$ » répond aux nécessités politiques de sa lutte avec Jean de Montfort, elle témoigne surtout de l'importation en Bretagne des pratiques capétiennes. Il convient en effet de rappeler à ce propos le rôle de la maison de France dans la réussite de la procédure canonique. Philippe VI, la reine, l'université de Paris et de nombreux évêques français ont apporté un soutien important et efficace à la cause du recteur de Trédrez auprès du souverain pontife. Comme d'autres figures du sanctoral français du XIIIe siècle (saint Yves en est, avec les saints Guillaume et Philippe de Bourges et saint Louis, une des les plus marquantes), l'official de Tréguier doit en partie son accession aux honneurs des autels à l'activité diplomatique de la maison de France ou de familles apparentées ${ }^{54}$. C'est donc un statut paradoxal que possède le personnage de saint Yves au sortir de la guerre de Succession. C'est en tant que saint breton (mais pour une part seulement) qu'il bénéficie de l'attachement du prétendant malheureux au trône ducal ; mais c'est grâce au soutien français (soutien d'abord antérieur aux querelles de la Succession de Bretagne) qu'il accédera finalement à la sanctification.

La construction du portail saint Yves de la cathédrale de Nantes constitue un exemple particulièrement frappant de cette volonté de récupération ou de captation de la part de Jean $\mathrm{V}$. En tout point ce cycle sculpté est remarquable : un siècle - peut-être très exactement ${ }^{55}$ après la canonisation de l'official de Tréguier ; dans la cathédrale la plus à l'est du duché ; à la porte de l'édifice la plus éminemment liée au pouvoir ducal, ce premier grand cycle monumental de saint Yves a manifestement pour but de "faire capétien" pourrait-on dire pour simplifier à l'extrême. La question de l'utilisation de la figure de saint Yves par Jean V se pose, on le voit donc, sous des dehors plus complexes qu'on ne pouvait le croire de prime abord. Plutôt qu'un cycle à vocation interne, visant à affirmer uniquement la domination

${ }^{53}$ CARON (Marie-Thérèse), Noblesse et pouvoir royal ..., p. 102.

${ }^{54}$ VAUCHEZ (André), La sainteté en occident..., p. 265.

${ }^{55}$ Les documents ne permettent pas d'être absolument définitif sur la date de ce cycle. Cependant, en fonction de la datation que nous proposons pour l'ensemble des portails de cette façade, la fourchette 1440-1460 peut être retenue. Les travaux ayant vraisemblablement commencé dans l'angle sud de la façade, il est tout à fait possible que la sculpture du portail saint Yves ait été en cours de conception, sinon d'élaboration en 1447. Nous 
Jean-Marie Guillouët, «L'iconographie de saint Yves et la politique dynastique des ducs Montfort à la fin du Moyen Âge » ("Saint Yves' Iconografy and Dynastic Strategy in Late Medieval Britany »), in Annales de Bretagne et des Pays de l'Ouest, t. 107, 2000, pp. 23-40. ducale sur le duché via un personnage emblématique de l'identité bretonne ${ }^{56}$, on verra dans les voussures nantaises un système de références exogènes, tirant ses sources et sa légitimité hors du duché, dans le domaine français. C'est donc plus en tant que figure "française" qu'en saint "breton" qu’Yves de Kermartin trouve sa place dans les voussures nantaises.

L'importance politique que revêt le culte de saint Yves au sein des querelles successorales du milieu du XIVe siècle ne disparaît donc pas au siècle suivant et l'enjeu que constitue encore au XVe siècle l'appropriation de la figure du saint de Kermartin apparaît clairement dans un épisode dramatique de la vie de Jean V. En 1420, alors que le duc croit se rendre à une conférence de conciliation avec les Penthièvre, il est enlevé à Châteauceaux par Olivier de Blois, comte de Penthièvre, petit-fils de Charles de Blois, poussé par Marguerite de Clisson, sa mère, et le dauphin Charles. On a prêté beaucoup de conséquences à cet attentat ; l'événement, il est vrai, fit grand bruit et marqua considérablement le prince breton ${ }^{57}$. Lors de cette captivité qui dura plusieurs semaines le duc promit à saint Yves pour sa délivrance son poids en or pour le tombeau du saint à Tréguier. Le monument, construit certainement dans la décennie 1420, ne nous est malheureusement plus connu que par des descriptions anciennes et imprécises ${ }^{58}$. Témoin de l'importance nouvelle que revêt le saint de Kermartin aux yeux du duc, le tombeau supportait selon Lobineau des bas reliefs illustrant « une partie des victoires de Jean le Conquérant, père de Jean $\mathrm{V}$, comme pour marquer la reconnaissance dont le père avoit chargé le fils de laisser des témoignages publics ${ }^{59} \gg$. La récupération du culte du saint par les Montfort à des fins politiques (sur un modèle, on le voit, proche de celui inauguré par Charles de Blois) s'illustre ici pleinement.

espérons pouvoir fournir une chronologie plus serrée de ces travaux à l'occasion de notre doctorat en cours à Paris IV sous la direction de Fabienne Joubert sur la sculpture de la façade de la cathédrale de Nantes.

${ }^{56}$ Si elle n'est peut-être pas totalement absente des voussures du portail Saint-Yves de la façade de SaintPierre, cette identification Bretagne-saint Yves est, nous l'avons souligné, suffisamment discrète voire absente des sources contemporaines pour faire la place à une autre interprétation. Il convient cependant de rester nuancé, le décor architectural par exemple réintroduit discrètement la référence bretonne dans ce portail.

${ }^{57}$ Le récit le plus détaillé et le plus complet de ces semaines mouvementées est encore celui qu'en a fait l'abbé Bourdeaut, il y a maintenant plus de 80 ans ! (BOURDEAUT (abbé), « Jean V et Marguerite de Clisson. La ruine de Châteauceaux », dans Bulletin de la Société Archéologique et Historique de Nantes et du département de la Loire-Inférieure, 1913, t. 54, pp. 331-417.

${ }^{58}$ On consultera sans s'attarder à la restitution proposée le texte de LA BORDERIE (A.), Rétablissement $d u$ tombeau de saint Yves. Notes pour les artistes bretons, Saint-Brieuc, Prud'Homme, 1885. 
Jean-Marie Guillouët, «L'iconographie de saint Yves et la politique dynastique des ducs Montfort à la fin du Moyen Âge » ("Saint Yves' Iconografy and Dynastic Strategy in Late Medieval Britany »), in Annales de Bretagne et des Pays de l'Ouest, t. 107, 2000, pp. 23-40.

Olivier de Blois de son côté semble avoir été parfaitement conscient de ces enjeux comme l'indique un épisode particulièrement dramatique de la captivité de Jean V raconté par le duc lui-même : «[...] nous dist iceluy Olivier qu'il avoit ouy que nos gens avoient assiegé Guingamp, \& quand nous estions allés-là en ce pays en pelerinage à Saint Yves; que ce n'avoit esté par dévotion, ains par hypocrisie ; \& que ce n'estoit que pour tourner avec nous ses hommes et subjets, \& qu'il sçavoit que le commun nous aimoit fort en celuy pays ; \& se doutoit bien que tantost ils rendroient sa ville de Guingamp ${ }^{60}$. » Cette anecdote trahit bien l'importance que revêt la figure de saint Yves dans un contexte de crise du pouvoir. Par ailleurs, loin d'avoir été la conséquence de l'attentat de Châteauceaux, la construction du tombeau de saint Yves à Tréguier pourrait en avoir été indirectement la cause. C'est en effet dès 1419 que Jean $\mathrm{V}$ prend contact avec Jacques de Hongrie, scolastique de Tréguier, pour construire sur le lieu de sépulture du saint un tombeau grandiose. Cette initiative, antérieure à l'attentat des Penthièvre, dont le « caractère provocateur peut contribuer à éclairer ce dernier événement ${ }^{61} »$, place bien l'appropriation de la figure du recteur de Trédrez au centre des enjeux politiques de ce premier tiers du XVe siècle.

\section{Une géographie politique}

Un regard plus attentif à ce cycle sculpté et aux groupes qui le composent permet de saisir plus clairement encore la volonté politique de Jean V. Il est en premier lieu frappant de constater l'absence à peu près complète de scènes judiciaires dans les voussures du portail nantais. L'iconographie de saint Yves semble pourtant avoir été largement diffusée sous sa forme judiciaire : le saint entre les deux plaideurs, le riche et le pauvre ${ }^{62}$. André Vauchez souligne à ce propos combien les réticences de la hiérarchie catholique de la fin du Moyen Âge à reconnaître la sainteté d'un simple prêtre avaient pu avoir pour conséquence la mise en valeur de la fonction de juge et d'avocat du personnage. Pour André Vauchez, l'iconographie «ne relève pas son rôle pastoral et son existence au milieu des paysans qui pourtant occupe

\footnotetext{
${ }^{59}$ LOBINEAU, Vie des saints de Bretagne, Rennes, 1724, p. 257 ; cité par LA BORDERIE, Rétablissement...,

${ }^{60}$ MORICE, Preuves..., t. II, col. 1076.

${ }^{61}$ Copy (Jean-Yves), Art, société et politique..., p. 185.

${ }^{62}$ Quelques représentations de saint Yves sont réunies dans MASSERON (Alexandre), Saint Yves, coll. « 1'art et les saints », Paris, [s.d.]; on y retrouvera essentiellement cette iconographie. Sur saint Yves avocat, voir JАСОВ (Robert), Les images de la justice : essai sur l'iconographie judiciaire du Moyen Âge à l'âge classique, « Le Léopard d’Or », Paris, 1994, p. 107-110.
} p. 10 . 
Jean-Marie Guillouët, «L'iconographie de saint Yves et la politique dynastique des ducs Montfort à la fin du Moyen Âge » (« Saint Yves' Iconografy and Dynastic Strategy in Late Medieval Britany »), in Annales de Bretagne et des Pays de l'Ouest, t. 107, 2000, pp. 23-40. tant de place dans les dépositions [du procès de canonisation] ${ }^{63} \gg$. Ce constat est tout à fait valable pour l'écrasante majorité des représentations de saint Yves ${ }^{64}$. Il ne se vérifie pas à Nantes cependant puisque, au contraire, les voussures qui lui sont consacrées font une large place à l'activité pastorale du saint et aux épisodes significatifs de son ascèse. Seules les scènes 20, 21 et 22 (épisode de la discorde et de la réconciliation d'une mère et d'un fils) peuvent avoir un lien avec le statut d'avocat du recteur de Trédrez; rapport on en conviendra assez indirect ${ }^{65}$.

A l'inverse, et de manière significative, le cycle insiste sur les scènes d'hommages et de supplications au tombeau du saint puisque trois groupes au moins (peut-être cinq) sont consacrés à ce thème pourtant formellement très répétitif. Une telle insistance a manifestement pour fonction de rappeler et de magnifier la construction du tombeau du saint à Tréguier par Jean V à partir de 1419-1420. Un jeu de correspondance s'établit ici entre les deux grands cycles sculptés de saint Yves dans le duché dans un raccourci géographique et politique fort significatif ${ }^{66}$. Le vingt-cinquième groupe décrit par Rousteau représente une des trois scènes conservées de supplication au tombeau. Le tombeau y est représenté de manière extrêmement minutieuse et détaillée, eut égard à la taille réduite de la sculpture, puisqu'on y distingue le soubassement d'arcades aveugles ainsi que le gisant, les mains jointes, surmonté d'un dais sculpté ${ }^{67}$. L'attrait suscité par la médiation miraculeuse du saint dans le duché est évoqué très clairement ici par la présentation, au-dessus du tombeau et des pèlerins, d'une poutre ou solive sur laquelle sont accrochés les ex-voto laissés par les fidèles (on y distingue une maison et peut-être un «chef» en cire). Toute cette attention portée, jusque dans les détails les plus étroits, à la réalité historique du culte rendu à saint Yves par les Bretons ne peut se comprendre en dehors du contexte qui vient d'être évoqué ici.

${ }^{63}$ VAUCHEZ (André), La sainteté en Occident..., p. 363.

64 Essentiellement mobiliaires (tableaux, panneaux et sculptures), elles appartiennent le plus souvent d'ailleurs à l'époque moderne.

${ }^{65}$ L'identification de ces trois scènes n'est, par ailleurs, pas certaine.

${ }^{66}$ Jean V, mort près de Nantes le 28 août 1442, sera d'ailleurs inhumé dans un premier temps dans la cathédrale de Nantes, les chaleurs de l'été empêchant semble-t-il son transport à Tréguier où l'attendait pourtant son tombeau, avant d'être transféré (après de nombreuses difficultés) ad sanctos, près de la sépulture de 1'official.

${ }^{67}$ Il convient de ne pas être dupe de cette représentation et de ne pas voir là une image du vrai tombeau de saint Yves à Tréguier. 
Ainsi, un cycle sculpté tel que celui consacré à saint Yves dans les voussures du portail sud de la cathédrale de Nantes permet de saisir la complexité des enjeux politiques entourant, à la fin du Moyen Âge, la figure de l'official de Tréguier. La manifestation d'influences bretonnes dans la décoration architecturale de ce portail et le lien étroit qu'il entretient avec le pouvoir temporel confèrent à l'iconographie de ses voussures une grande importance. Traditionnellement interprété avant tout comme un saint breton (ce qui ne semble être attesté qu'à partir de l'époque moderne), saint Yves doit cependant être reconsidéré en fonction du contexte très particulier qui l'a vu s'élever à la dignité des autels. La guerre de succession de Bretagne, la compétition Montfort-Penthièvre et singulièrement l'action de Charles de Blois, le compétiteur malheureux de Jean de Montfort, ont conféré à l'imagerie du saint de Kermartin un statut politique tout à fait spécifique. Au même titre que la couronnne fleuronnée ou la titulature, l'iconographie de saint Yves traduit l'importation en Bretagne d'habitudes et de pratiques monarchiques capétiennes qui révèleront toute leur importance à la fin du XVe siècle lors des crispations diplomatiques avec le royaume de France. Il fallait que saint Yves fût passé par le singulier détour de la politique capétienne pour que Jean $\mathrm{V}$ tentât d'en faire par la suite un saint breton. 